\title{
Super Stereoscopy Technique for Comfortable and Realistic 3D Displays
}

\author{
Kaan Akşit, Amir Hossein Ghanbari Niaki, Erdem Ulusoy, Hakan Urey \\ Koç University, Electrical Engineering Department and Optical Microsystems Laboratory, Istanbul, Turkey \\ *Corresponding author: hurey@ku.edu.tr \\ Received Month X, XXXX; revised Month X, XXXX; accepted Month X, XXXX; \\ posted Month X, XXXX (Doc. ID XXXXX); published Month X, XXXX
}

\begin{abstract}
Two well-known problems of stereoscopic displays are the accommodation-convergence conflict and the lack of natural blur for defocused objects. We present a new technique that we name Super Stereoscopy (SS3D) to provide a convenient solution to these problems. Regular stereoscopic glasses are replaced by SS3D glasses which deliver at least two parallax images per eye through pinholes equipped with light selective filters. The pinholes generate blur free retinal images so as to enable correct accommodation, while the delivery of multiple parallax images per eye creates an approximate blur effect for defocused objects. Experiments performed with cameras and human viewers indicate that the technique works as desired. In case two pinholes equipped with color filters per eye are used, the technique can be used on a regular stereoscopic display by only uploading a new content, without requiring any change in display hardware, driver or frame rate. Apart from some tolerable loss in display brightness and decrease in natural spatial resolution limit of eye due to pinholes, the technique is quite promising for comfortable and realistic $3 D$ vision, especially enabling the display of close by objects that are not possible to display and comfortably view on regular 3DTV and cinema.

OCIS Codes:
\end{abstract}

Stereoscopic three dimensional (3D) displays generally invoke 3D perception only through the binocular disparity cue by providing a pair of parallax images to two eyes [1, 2 ]. The images appear sharp only when the eyes focus on the display screen. Thus, though the eyes converge truly to the apparent position of a virtual object, they accommodate incorrectly on the screen rather than the virtual object plane to make the retinal images free of blur, as illustrated in Fig.1a and Fig.1b for an exemplary stereoscopic system that uses polarization encoding for separation of left and right views. This well-known accommodation-convergence $(\mathrm{A} / \mathrm{C})$ conflict causes viewing discomfort for 3DTV and cinema and becomes a major problem when the virtual objects are closer than $0.5 \mathrm{~m}[3$, 4]. In addition, regardless of their depth, all objects appear sharp simultaneously in conventional stereoscopic displays. The natural blur of defocused objects is absent. In this work, we introduce a novel technique that we name Super Stereoscopy (SS3D), which we first briefly introduced in [5] and which resolves these problems of conventional stereoscopic displays with quite minimal modifications over the existing systems.

In SS3D, we replace the regular stereoscopic glasses with new glasses on which pinholes that are sufficiently smaller than the pupil of the eye are placed. Images entering the eye through these pinholes are almost always sharp, regardless of the accommodation distance of the eyes. Then, the accommodation of the eyes is governed by the binocular disparity and convergence cues only, but not by image blur, enabling accommodation on the object [4]. Hence, accommodation response is corrected and the $\mathrm{A} / \mathrm{C}$ conflict is resolved. A single pinhole per each eye would be sufficient for this purpose. But then, the absence of natural blur for defocused objects persists. To also resolve this issue, we place two pinholes equipped with different light selective filters and deliver two different parallax images per eye. For a focused virtual object, these two images completely overlap and merge into a single sharp retinal image, while in the case of a defocused object; the two parallax images form two sharp but slightly shifted retinal images, as illustrated in Fig.1c and Fig.1d for an exemplary SS3D system in which left and right views are separated by polarization encoding, and two views per each eye are separated by red and cyan color filters. Hence, the retinal image of a defocused object, though not perfect, is closer to being natural. Actually, if more than two pinholes and parallax images per eye are used, the number of slightly shifted retinal images for a defocused object can be increased and even more natural blurring effect can be achieved. However, this case requires glasses and high frame rate displays that use sophisticated color, polarization, and temporal multiplexing schemes. Content generation and delivery routines also get complicated. On the other hand, quite easy to implement options are available for the case of two parallax images per eye. In particular, if the SS3D system depicted in Fig.1 (or a similar version where left and right views are separated by shutter based glasses rather than polarized glasses) is used, a regular stereoscopic display (that uses polarization or temporal multiplexing) can be used in SS3D mode as well, without any hardware or driver modification. Merely, each frame of SS3D content (4 images, 2 per eye) should be color coded into a frame of the regular stereoscopic content ( 2 images, 1 per eye) that is fed to the display. This case, demonstrated in our prototype and further detailed below, provides an easy extension possibility for regular stereoscopic 3DTV and cinema into SS3D. 


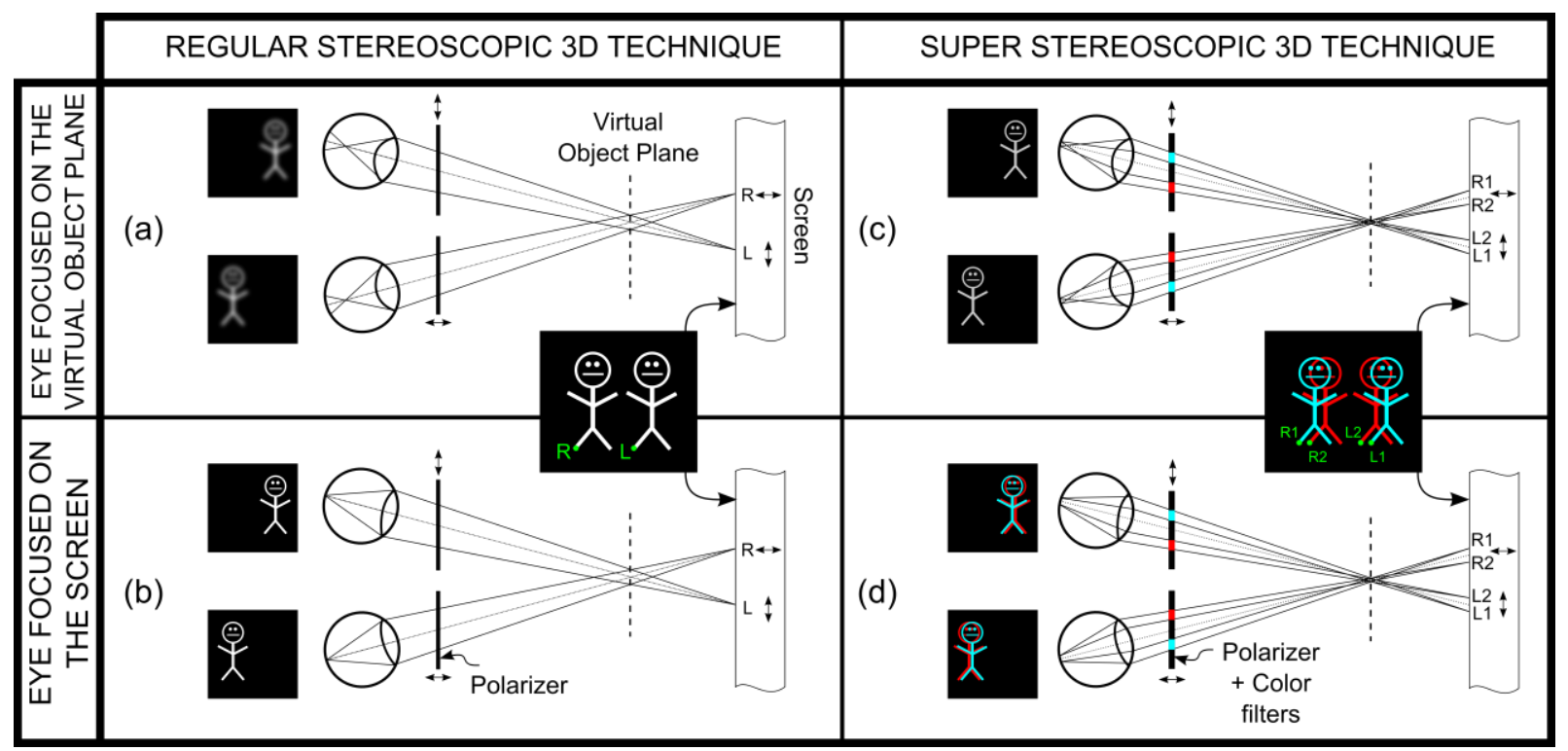

Figure 1.Retinal image formations in regular stereoscopic 3D and SS3D systems. In regular stereoscopic 3D displays, eyes converge on the virtual object. However they cannot stay focused on virtual object plane since in that case retinal images are blurred as in (a), so they change their focus to the screen in order to sharpen retinal images as in (b), resulting in $\mathrm{A} / \mathrm{C}$ conflict. In $\mathrm{SS} 3 \mathrm{D}$, the pinholes lead to retinal images which are always sharp, hence eyes can stay focused on the virtual object plane, as in (c), eliminating the $\mathrm{A} / \mathrm{C}$ conflict. In fact, when eyes are focus on a different plane, parallax images get separated from each other, as in (d), providing an approximate blur effect.

Fig. 2 is the experimental demonstration of SS3D concept for a single eye using an image on an LCD screen placed at $60 \mathrm{~cm}$ away from a camera. In front of the camera, we place two pinholes. The pinholes used for this experiment have a diameter of $1 \mathrm{~mm}$ and the distance between pinhole centers is $2 \mathrm{~mm}$. The left and right pinholes are equipped with red and cyan color filters, respectively. Three sets of red and cyan bars (Fig. 2a) have disparities such that from top to bottom, three virtual white bars are supposed to appear at 35,50 and $60 \mathrm{~cm}$. When the camera is focused at three different depths, the images with no pinholes appear sharp only at the screen (Fig.2b) and are substantially blurred at other planes (Fig.2c\&2d), illustrating that in conventional stereoscopy it is not possible to achieve correct accommodation. Using the pinholes in front of the camera, each bar appears white and sharp at the corresponding virtual object plane, illustrating that in SS3D it is possible to achieve correct accommodation (Fig.2f\&2g). We also see the formation of double partially overlapping images in the case of pinholes when the accommodation is shifted away from the virtual object plane, illustrating that in SS3D we achieve more natural vision with an approximate blur effect.

Pinhole diameter, eye-relief distance, and pinhole separation are important design parameters of SS3D glasses. Pinhole diameter is the main determinant of voxel width, hence the ability of the system to trigger correct accommodation response. From a geometrical optics perspective, as the pinhole diameter is made smaller, the voxel width gets smaller and image blurring on the retina is reduced. However, from a wave optics perspective, as the pinhole diameter gets smaller, diffraction effects become predominant and as a consequence retinal images get blurred. Moreover, the captured light power decreases with shrinking pinholes. Therefore, there is an optimum size for the pinhole diameter. Our trial with different pinhole sizes indicate that about $0.6 \mathrm{~mm}$ diameter is a good choice for a screen placed around a meter.

Eye-relief and pinhole separation distances are the main determinants of field of view (FOV) of SS3D glasses. The FOV obtained using SS3D glasses is shown in Fig.3. As seen, there is a full FOV ( FOV $\left._{f}\right)$ where the image is seen through at least one of the pinholes, and there is the smaller super stereoscopic FOV $\left(\mathrm{FOV}_{\mathrm{ss}}\right)$ where the super

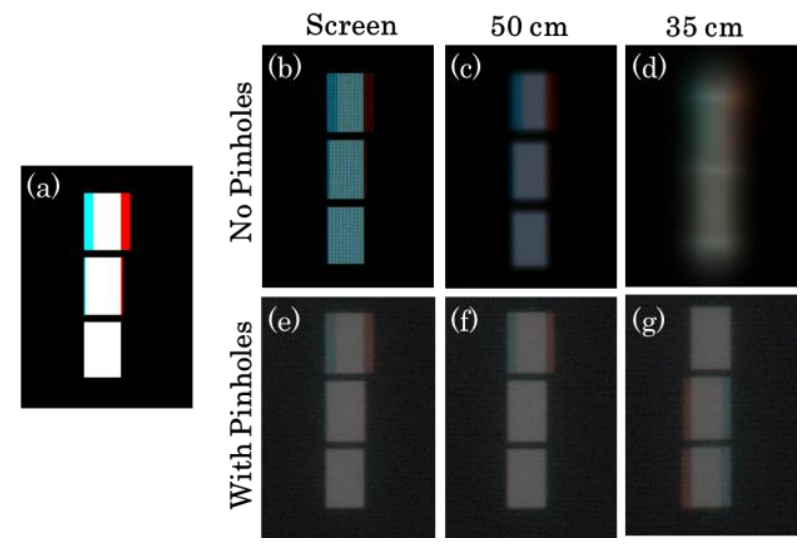

Figure 2. Camera-based experiment illustrating SS3D concept for one eye. (a) is the content shown on the screen. The camera is focused (b) on the screen without pinholes, (c) at $50 \mathrm{~cm}$ without pinholes, (d) at $35 \mathrm{~cm}$ without pinholes, (e) on the screen with pinholes, (f) at $50 \mathrm{~cm}$ with pinholes, (g) at $35 \mathrm{~cm}$ with pinholes. Images are enhanced for better visualization. 
stereoscopic image can be seen through both pinholes. It is easy to see from the figure that decreasing the eye relief increases both $\mathrm{FOV}_{\mathrm{f}}$ and $\mathrm{FOV}_{\mathrm{ss}}$, but obviously there is a lower limit to this since a too close eyeglass will be disturbing to the user. On the other hand, increasing pinhole separation increases $\mathrm{FOV}_{\mathrm{f}}$ but decreases $\mathrm{FOV}_{\mathrm{ss}}$, hence a compromise can be made here depending on the application, as long as the screen is included in FOV $\mathrm{Vss}_{\text {. }}$ Finally, note that due to the presence of pinholes in front of the eye, the FOV of a single naked eye (which is about 95-115 degrees in horizontal) is somewhat decreased. However, this is not a significant reduction when typical applications are considered. For instance, for a viewer standing $1 \mathrm{~m}$ away from a 20 " display, the used portion of FOV is only about 30 degrees, which can easily be provided with SS3D glasses with reasonable selections of eye relief and pinhole separation distances.

The pinhole separation is also the main determinant of the amount of artificial blurring introduced for defocused objects. If the pinholes are too close to each other, the parallax images overlap on the retina for a greater range of distances, so the blur effect is weak. On the other hand, if the pinholes are placed too widely, the retinal images get separated too quickly, giving the impression of double vision rather than blur. Therefore, there is also a tradeoff here, in addition to the FOV criterion. Our trials indicate that a pinhole separation of $1.4 \mathrm{~mm}$ for $5 \mathrm{~mm}$ eye relief is a good choice.

We built a prototype SS3D eyeglass to perform experimental tests in our lab. Fig.4b shows the picture of the prototype SS3D glasses. On both sides of the eyeglass, there are two pinholes with embedded color filters. The pinholes have a diameter of $0.6 \mathrm{~mm}$ and a separation of $1.4 \mathrm{~mm}$, with a red color filter on the left and a cyan color filter on the right (as seen from the viewer's side). The prototype eyeglass does not have any polarizers or shutters as it is tested using a 3D display system that is already capable of separating left and right views without requiring the viewer to wear any glasses. This $3 \mathrm{D}$ display system is also developed in our lab [6]. As illustrated in Fig. 4a, our 3D display uses two pico-projectors developed by Microvision Inc. and placed above the viewer's head. The projection screen contains a retro-reflective film and a vertical diffuser. The screen produces two vertical viewing slits for the left and right eyes. Viewer's head is tracked

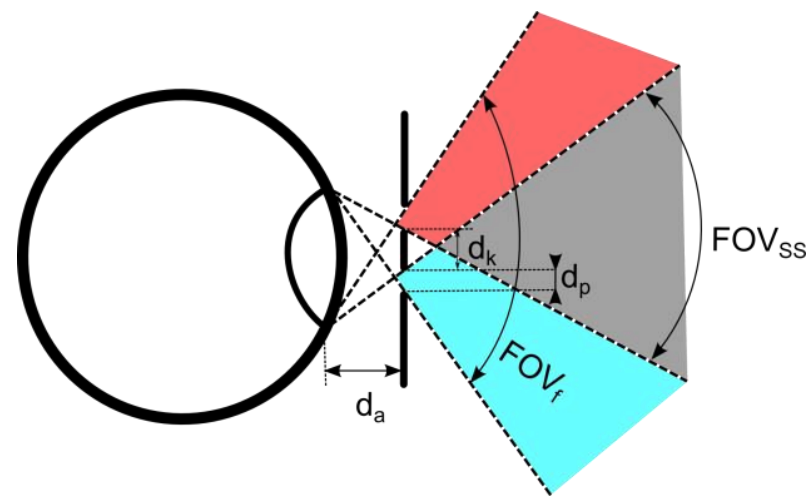

Figure 3.FOV for SS3D. $\mathrm{d}_{\mathrm{k}}$ is pinhole separation, $\mathrm{d}_{\mathrm{p}}$ is the pinhole diameter, and $\mathrm{d}_{\mathrm{a}}$ is the eye-relief distance.

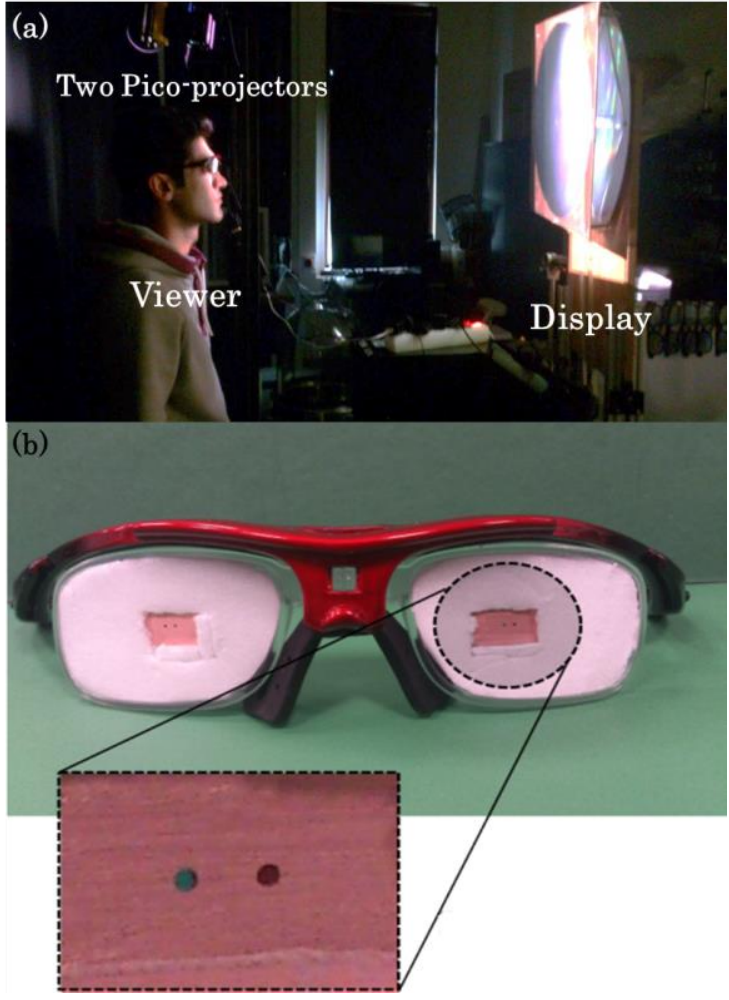

Figure 4.a) The setup used for testing, b) pinhole glass prototype with pinhole diameter of $0.6 \mathrm{~mm}$ and pinhole separation of $1.4 \mathrm{~mm}$.

with a camera and the viewing slits are automatically aligned with the viewer's eyes by rotating the screen, allowing head motion. If desired, the content can be updated in synchronism with head position to provide both horizontal and vertical motion parallax.

The content creation procedure is shown in Fig.5. Each frame of the display content consists of four parallax images of a single 3D scene (Fig. 5a). Two of these images are for the left eye, and the other two are for the right eye. For any of the eyes, the green and blue channels of the left pinhole's image and the red channel of the right pinhole's image are discarded (Fig.5b). The kept channels are superimposed to create an RGB image which is then fed to the associated pico-projector (Fig.5c).

As a preliminary test of the proposed technique, we performed an experiment with 15 subjects among students of Koç University. The subjects were not informed about the SS3D technique before the test. The content shown in Fig.5c was displayed on the system shown in Fig.4a. The screen was placed at $100 \mathrm{~cm}$ away from the viewing position, and the disparities are calculated such that the large pumpkin appears at $50 \mathrm{~cm}$, the goose is at $100 \mathrm{~cm}$ and the small pumpkin is at 115 $\mathrm{cm}$. The subjects were first asked to wear SS3D eyeglasses and align the glasses for their interpupillary distances such that pinholes are symmetrically aligned in front of each eye pupil. Secondly, they were asked to focus on their thumbs and move it to a position where the big pumpkin is also in focus. At that position, the distance between their thumb and the screen was measured. The 


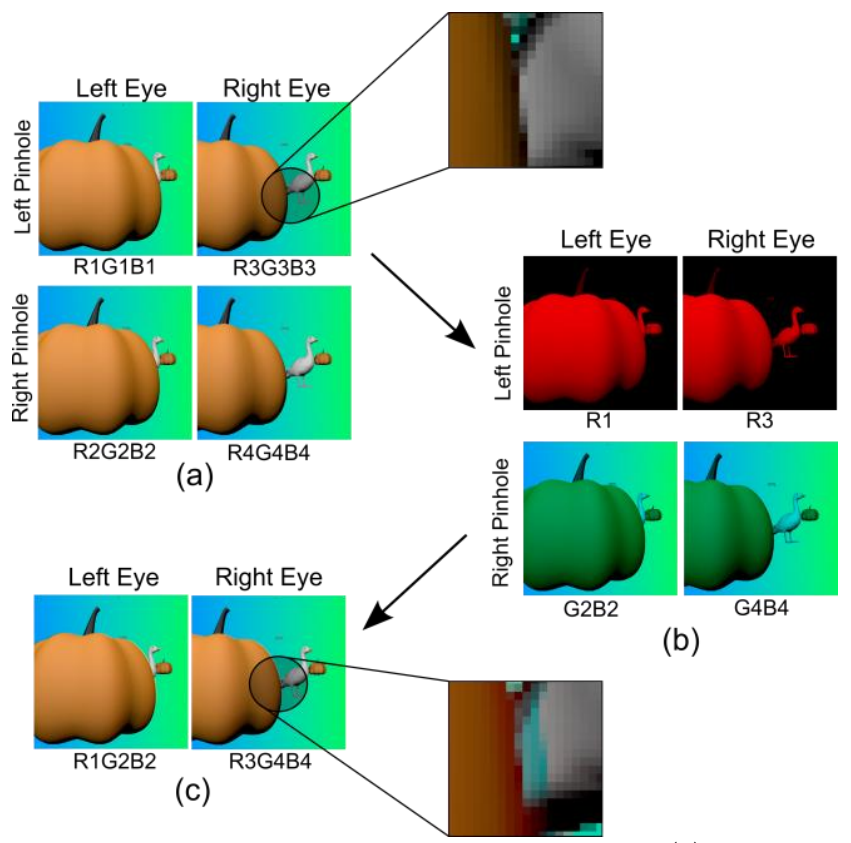

Figure 5. Content creation procedure. (a) Images exported directly from rendering software, b) images after discarding the relevant color channels, and c) final images obtained by superimposing the preserved channels. The magnified portion in (c) illustrates the color shifts formed due to superimposition of parallax images. No such color shift is present in the magnified portion of (a).

average of these measurements turned out to be $50.5 \mathrm{~cm}$ with a standard deviation of $15.7 \mathrm{~cm}$. This initial experiment and impressions of subjects indicate that SS3D technique seems promising for improving the 3D depth perception.

To conclude, we introduce in this paper the novel SS3D technique which provides quite convenient solutions to the $\mathrm{A} / \mathrm{C}$ conflict and lack of natural blur problems of conventional stereoscopic displays. In our view, the most important advantage of our technique is that it can be used with minor modifications over the existing stereoscopic systems. The particular eyeglass design that uses two pinholes with color filters on each side only requires a regular polarizer or shutter based stereoscopic display to work with, providing easy adaption possibility to existing 3DTV and cinema systems. Though we selected this case for our demonstrations, it is also possible to come up with convenient SS3D system designs that deliver higher number of parallax images for each eye. For instance, three parallax images can be provided per eye by using RGB color filters instead of red and cyan color filters. This strategy again can function using a conventional stereoscopic display. In another strategy, one can use a polarization multiplexing stereoscopic display in synchronism with an SS3D glass equipped with electronic shutters and two sets of RGB color filters on each side (giving six pinholes per eye) where the two sets are covered with complementary polarizers. In this manner, six parallax images can be delivered per eye at the cost of halved frame rate of the display, and so on. Actually, a similar idea to ours has been presented under the name
Super Multi View (SMV) displays [7, 8]. SMV displays are multi-view autostereoscopic displays that aim to provide a large number of viewing slits such that at least two of these slits are intercept by the eye at a time, with an intention to resolve the $\mathrm{A} / \mathrm{C}$ conflict. However, due to their multi-view and autostereoscopic nature, experimental demonstrations of SMV systems require quite complex hardware and show only limited success as the viewing slits produced at large distances from the display screen cannot be sufficiently small due to optical limitations. In addition, having vertical slits instead of pinholes degrade the ability to trigger correct accommodation. On the contrary, our technique is much easier to implement and demonstrate. Moreover, when supplemented with appropriate tracking and content update modules, our technique is also much easier to implement a multi-view system. Obviously, the main drawback of our technique is some loss in brightness and decrease in natural spatial resolution limit of the eye due to the usage of pinholes. However, we believe that these losses are within tolerable limits and the benefits of SS3D technique far outweigh them.

\section{References:}

1. H. Urey, K. V. Chellappan, E.Erden, and P.Surman. Proceedings of the IEEE99, no. 4 (2011): 540-555.

2. J. Geng. Advances in Optics and Photonics 5, no. 4 (2013): 456-535.

3. D. M. Hoffman, A. R. Girshick, K. Akeley, and M. S. Banks. Journal of Vision 8, no. 3 (2008): 33.

4. M. Lambooij, M.Fortuin, I.Heynderickx, and W.IJsselsteijn. Journal of Imaging Science and Technology53, no. 3 (2009): 30201-1.

5. Aksit, Kaan, Amir Hossein Ghanbari Niaki, Osman Eldes, and Hakan Urey. The True Vision-Capture, Transmission and Display of $3 D$ Video (3DTV-CON), 2014, pp. 1-3. IEEE, 2014.

6. O. Eldes, K.Akşit, and H. Urey. Optics Express 21, no. 23 (2013): 29043-29054.

7. K. Choi, H. Lee, Y. Choi, J. Bae, H. Song (2011). U.S. Patent Application 13/298,142.

8. Y. Takaki, K. Tanaka, and J. Nakamura. Optics Express 19, no. 5 (2011): 4129-4139. 
1. H. Urey, K. V. Chellappan, E.Erden, and P.Surman. "State of the art in stereoscopic and autostereoscopic displays." Proceedings of the IEEE99, no. 4 (2011): 540555 .

2. J. Geng. "Three-dimensional display technologies." Advances in Optics and Photonics 5, no. 4 (2013): 456-535.

3. D. M. Hoffman, A. R. Girshick, K. Akeley, and M. S. Banks. "Vergence-accommodation conflicts hinder visual performance and cause visual fatigue." Journal of vision 8, no. 3 (2008): 33.

4. M. Lambooij, M.Fortuin, I.Heynderickx, and W. IJsselsteijn. "Visual discomfort and visual fatigue of stereoscopic displays: a review." Journal of Imaging Science and Technology 53, no. 3 (2009): 30201-1.

5. Aksit, Kaan, Amir Hossein Ghanbari Niaki, Osman Eldes, and Hakan Urey. "Super stereoscopy 3D glasses for more realistic $3 \mathrm{D}$ vision." In $3 D T V$-Conference: The True Vision-Capture, Transmission and Display of $3 D$ Video (3DTV-CON), 2014, pp. 1-3. IEEE, 2014.

6. O. Eldes, K.Akşit, and H. Urey. "Multi-view autostereoscopic projection display using rotating screen." Optics Express 21, no. 23 (2013): 29043-29054.

7. K. Choi, H. Lee, Y. Choi, J.Bae, and H. Song. "3D display apparatus and method of displaying 3D images." U.S. Patent Application 13/298,142, filed November 16, 2011.

8. Y. Takaki, K. Tanaka, and J. Nakamura. "Super multiview display with a lower resolution flat-panel display." Optics Express 19, no. 5 (2011): 4129-4139. 